\title{
Associated production of Higgs at linear collider in the inert Higgs Doublet Model
}

\author{
Abdesslam Arhrib ${ }^{1,2, a}$, Rachid Benbrik ${ }^{3,4,5, b}$, Tzu-Chiang Yuan ${ }^{2, c}$ \\ ${ }^{1}$ Département de Mathématique, Faculté des Sciences et Techniques, Université Abdelmalek Essaadi, B. 416, Tangier, Morocco \\ ${ }^{2}$ Institute of Physics, Academia Sinica, Nankang, Taipei 11529, Taiwan \\ ${ }^{3}$ MSISM team, Département de Physique, Faculté Polydisciplinaire de Safi, Sidi Bouzid, B.P. 4162, 46000 Safi, Morocco \\ ${ }^{4}$ Instituto de Fisica de Cantabria (CSIC-UC), Santander, Spain \\ ${ }^{5}$ LPHEA, FSSM, Cadi Ayyad University, B.P. 2390, Marrakech, Morocco
}

Received: 10 February 2014 / Accepted: 5 May 2014 / Published online: 27 May 2014

(c) The Author(s) 2014. This article is published with open access at Springerlink.com

\begin{abstract}
We study the correlation between the Standard Model Higgs decay $h \rightarrow \gamma \gamma$ and $h \rightarrow Z \gamma$ in the Inert Higgs Doublet Model. It is found that these two one-loop-induced decays are positively correlated, with the latter channel having slightly smaller branching ratio than the former one. At the Linear Collider, we study the interplay of the off-shell extension of these two amplitudes that contributed significantly to the associated production of the Higgs boson with a photon in the process $e^{+} e^{-} \rightarrow \gamma h$ and with an electron in the process $e^{-} \gamma \rightarrow e^{-} h$ in the $s$ and $t$ channels, respectively, via both $\gamma$ and $Z$ exchange for each process.
\end{abstract}

\section{Introduction}

Recently the ATLAS and the CMS Collaborations using the combined $7 \oplus 8 \mathrm{TeV}$ data found a bosonic resonance with a mass around $125-126 \mathrm{GeV}$ in two photons, two $Z$ and two $W$ channels $[1,2]$. This discovery is also confirmed by the final result from Tevatron at CDF and D0 experiments through the associated production process $p \bar{p} \rightarrow$ $W h \rightarrow(l v)(b \bar{b})$ [3]. The new particle is necessarily a boson, since it decays into two photons, two $Z$ and two $W$ bosons, and it could possibly be the missing particle of the Standard Model (SM), the Brout-Englert-Higgs boson $h$. For this Higgs-like particle, ATLAS obtained its mass of $125.5 \pm 0.2$ (stat.) ${ }_{-0.6}^{+0.5}$ (syst.) $\mathrm{GeV}$ [4], while CMS got $125.7 \pm 0.3$ (stat.) \pm 0.3 (syst.) $\mathrm{GeV}[5]$. At the Moriond and EPS conferences, ATLAS and CMS updated their results on

\footnotetext{
a e-mail: aarhrib@ictp.it

b e-mail: rbenbrik@ictp.it

c e-mail: tcyuan@phys.sinica.edu.tw
}

$h \rightarrow \gamma \gamma, Z Z, W W, \tau \tau$ and $b \bar{b}$ channels with an integrated luminosity of up to $5 \mathrm{fb}^{-1}$ at $7 \mathrm{TeV}$ and up to $21 \mathrm{fb}^{-1}$ at 8 $\mathrm{TeV}$. For ATLAS the combined signal strength is found to be $\mu=1.23 \pm 0.18$ at the new combined mass measurement $[6,7]$. For the CMS update, the combined strength is found to be $\mu=0.8 \pm 0.14[5,8]$. All these latest experimental developments led to great excitement by the announcement of the 2013 Nobel Prize of Physics being awarded to F. Englert and P. W. Higgs due to their seminal works $[9,10]$ five decades ago.

Since the new particle decays to pairs of gauge bosons and fermions, a non-integer spin is already ruled out. According to the Landau-Yang theorem $[11,12]$, given the fact that the new boson decays into pair of photons, it excludes the spin 1 possibility and then the remaining possibility is either 0 or 2. Recently, spin and parity of the Higgs-like particle were studied from the angular distributions of the diphoton, $Z Z^{*}$ and $W W^{*}$ decay channels [13-17] at ATLAS and CMS by looking at the kinematical information of the final states: photons and leptons. Both collaborations disfavor the pure pseudoscalar or spin-2 hypotheses. In the case of the disfavored CP-odd Higgs with $J^{P C}=0^{-+}$its branching ratio into a pair of $W \mathrm{~s}$ or $Z \mathrm{~s}$ is expected to be two orders of magnitude smaller than the observed one. From these analysis, the spin-1 hypothesis is also disfavored with an even higher confidence level.

In order to further validate the Higgs mechanism of mass generation in the SM, one still need to establish the following measurements with high precision: (1) the spin of the Higgs boson, (2) its $C P$ quantum number, (3) its couplings to fermions and to gauge bosons, and (4) the triple and quartic self-couplings of the Higgs boson.

After more than two decades of studies, technical design report for the International Linear Collider (ILC) has now 
been completed (see the Technical Design Report [18,19] for details). Indeed, detailed simulations for various physical cases with realistic detector properties show that the ILC can achieve impressive precision measurements for Higgs and top quark physics [20,21]. The ILC [22] program will be running for center-of-mass energies between 200 and 500 $\mathrm{GeV}$, with rapid changes in energy to allow for threshold scans such as $Z h$ at $250 \mathrm{GeV}, t \bar{t}$ at $350 \mathrm{GeV}$, as well as $Z h h$ and $t \bar{t} h$ at $500 \mathrm{GeV}$. Ultimately, increasing the ILC centerof-mass energy to $1 \mathrm{TeV}$ is also envisioned.

Clearly, first run of the $\mathrm{LHC}$ at $7 \oplus 8 \mathrm{TeV}$ has initiated the first step of a precise measurement program for Higgs physics which will get improved at the LHC $13-14 \mathrm{TeV}$ run with more data accumulated. It is well known that the precise measurement programs at the ILC and LHC are complementary to each other in many aspects [20,23]. Options of $\gamma \gamma$ and $e^{-} \gamma$ collisions at the ILC provide an unique opportunity for precise measurements for Higgs properties. Thus ILC can yield substantial improvements over LHC measurements. Moreover, ILC will have great advantage in terms of the quality of signatures of new physics, which may be overwhelmed by huge QCD backgrounds at the hadronic environment of LHC.

The extraction of the Higgs-like couplings to gauge bosons and fermions achieved up to now from the $7 \oplus 8 \mathrm{TeV}$ data shows that this new boson behaves more and more like a SM Higgs boson [5-8]. More data is needed in order to fully pin down the exact nature of the newly discovered particle. The fact that the Higgs-like particle couplings to gauge bosons and fermions are consistent with SM prediction can put severe constraints on all models extending the SM that try to accommodate such a Higgs-like particle.

As we now know, the loop-induced process $h \rightarrow \gamma \gamma$ turns out to be a discovery mode for the $125-126 \mathrm{GeV}$ Higgs using the existing LHC data. The other related loop-induced decay $h \rightarrow Z \gamma$ has not been seen yet but is expected to be measured at the future LHC $13-14 \mathrm{TeV}$ run when more data is accumulated. Any additional charged particles beyond those in the SM will contribute to the loop amplitudes for these two processes. Thus it is important to measure these two modes as accurate as possible. An alternative way to extract the $h \gamma \gamma$ and $h Z \gamma$ couplings is to study the associated production of $e^{+} e^{-} \rightarrow \gamma^{*}, Z^{*} \rightarrow \gamma h$ at the ILC. Fusion production of $\gamma \gamma \rightarrow h$ and associated production with an electron via $e^{-} \gamma \rightarrow e^{-} h$ are also interesting to study if these options of $\gamma \gamma$ and $e^{-} \gamma$ collisions are available at the ILC.

In this paper, we concentrate on the Inert Higgs Doublet Model (IHDM) which is basically a two Higgs Doublet Model (THDM) with an exact $\mathbb{Z}_{2}$ symmetry imposed. Under the $\mathbb{Z}_{2}$ symmetry, all the SM particles are even and only the second Higgs doublet is odd. The model was first proposed by Deshpande and Ma [24] to study the pattern of electroweak symmetry breaking. Much later, it was extended further as a model of scalar dark matter together with a radiative seesaw mechanism of neutrino mass [25].

We organize this paper as follows. In Sect. 2, we briefly review IHDM to set up our notations and mention some theoretical and experimental constraints for the model. We discuss the correlation of the signal strengths for the two loopinduced processes $h \rightarrow \gamma \gamma$ and $h \rightarrow Z \gamma$ in IHDM in Sect. 3 . We study the two processes $e^{+} e^{-} \rightarrow \gamma h$ and $e^{-} \gamma \rightarrow h e^{-}$ in IHDM at the ILC in Sect. 4. We conclude in Sect. 5.

\section{The inert Higgs Doublet Model}

Besides the SM Higgs doublet $H_{1}$, the IHDM [24] employs an additional Higgs doublet $H_{2}$, which can be parameterized as follows:

$$
\begin{aligned}
& H_{1}=\left(\begin{array}{c}
G^{+} \\
v / \sqrt{2}+\left(h+i G^{0}\right) / \sqrt{2}
\end{array}\right), \\
& H_{2}=\left(\begin{array}{c}
H^{+} \\
(S+i A) / \sqrt{2}
\end{array}\right)
\end{aligned}
$$

where $G^{ \pm}$and $G^{0}$ are the charged and neutral goldstone bosons. IHDM imposes a discrete $\mathbb{Z}_{2}$ symmetry under which all the SM fields and $H_{1}$ are even while $H_{2}$ is odd. The scalar potential allowed by the $\mathbb{Z}_{2}$ symmetry is given by

$$
\begin{aligned}
V= & \mu_{1}^{2}\left|H_{1}\right|^{2}+\mu_{2}^{2}\left|H_{2}\right|^{2}+\lambda_{1}\left|H_{1}\right|^{4}+\lambda_{2}\left|H_{2}\right|^{4} \\
& +\lambda_{3}\left|H_{1}\right|^{2}\left|H_{2}\right|^{2}+\lambda_{4}\left|H_{1}^{\dagger} H_{2}\right|^{2} \\
& +\frac{\lambda_{5}}{2}\left\{\left(H_{1}^{\dagger} H_{2}\right)^{2}+\text { h.c. }\right\} .
\end{aligned}
$$

The electroweak gauge symmetry is broken when $H_{1}$ develops its vacuum expectation value (VEV) $\left\langle H_{1}\right\rangle^{\mathrm{T}}=$ $(0, v / \sqrt{2})$, while $\left\langle H_{2}\right\rangle=0$ to maintain the $\mathbb{Z}_{2}$ symmetry so as to allow for a dark matter (DM) candidate in this inert doublet. This pattern of symmetry breaking results in two CP-even neutral scalars $(h, S)$, one CP-odd neutral scalar $(A)$, and a pair of charged scalars $\left(H^{ \pm}\right)$. Note that $h$ is the SM Higgs and is $\mathbb{Z}_{2}$-even, while $S, A$ and $H^{ \pm}$are $\mathbb{Z}_{2}$-odd. Only SM Higgs $h$ couples to SM fermions, while $S, A$ and $H^{ \pm}$are inert and do not couple to fermions. The lighter one of the two scalars $S$ or $A$ can be a cold dark matter candidate in IHDM. In what follows, we will denote by $\chi$ the DM candidate in this model, whether it is $S$ or $A$. Many phenomenological aspects of dark matter physics in IHDM have been studied over the years [26-29]. For an updated global analysis of IHDM, we redirect our readers to Ref. [30] where extensive references of previous works can be found as well.

The masses of the four physical scalars can be written in terms of the parameters $\mu_{2}^{2}$ and $\lambda_{i}(i=1,3,4,5)$ as 


$$
\begin{aligned}
& m_{h}^{2}=-2 \mu_{1}^{2}=2 \lambda_{1} v^{2}, \\
& m_{S}^{2}=\mu_{2}^{2}+\frac{1}{2}\left(\lambda_{3}+\lambda_{4}+\lambda_{5}\right) v^{2}=\mu_{2}^{2}+\lambda_{L} v^{2}, \\
& m_{A}^{2}=\mu_{2}^{2}+\frac{1}{2}\left(\lambda_{3}+\lambda_{4}-\lambda_{5}\right) v^{2}, \\
& m_{H^{ \pm}}^{2}=\mu_{2}^{2}+\frac{1}{2} \lambda_{3} v^{2},
\end{aligned}
$$

where we have defined $\lambda_{L} \equiv \frac{1}{2}\left(\lambda_{3}+\lambda_{4}+\lambda_{5}\right)$ for later convenience. One can also invert the above relations to write the quartic coupling $\lambda_{i}(i=1,3,4,5)$ in favor of the four physical scalar masses and the parameter $\mu_{2}^{2}$,

$$
\begin{aligned}
\left\{\lambda_{1}, \lambda_{3}, \lambda_{4}, \lambda_{5}\right\}=\frac{1}{v^{2}}\left\{\frac{m_{h}^{2}}{2}, 2\left(m_{H^{ \pm}}^{2}-\mu_{2}^{2}\right),\right. & \left(m_{S}^{2}+m_{A}^{2}-2 m_{H^{ \pm}}^{2}\right), \\
& \left.\left(m_{S}^{2}-m_{A}^{2}\right)\right\} .
\end{aligned}
$$

In our numerical study presented in the next two sections, we will choose the following set of parameters:

$\mathcal{P}=\left\{m_{h}, m_{S}, m_{A}, m_{H^{ \pm}}, \lambda_{2}, \mu_{2}^{2}\right\}$

to fully describe the scalar sector of IHDM.

\subsection{Theoretical and experimental constraints}

The parameter space of IHDM discussed above is subjected to both theoretical and experimental constraints as we will describe briefly here.

- Inert vacuum: In order to realize the inert vacuum described earlier, one must have [31]:

$$
m_{h}^{2}, m_{H}^{2}, m_{A}^{2}, m_{H^{ \pm}}^{2}>0 \text { and } \mu_{1}^{2} / \sqrt{\lambda_{1}}<\mu_{2}^{2} / \sqrt{\lambda_{2}}
$$

- Perturbativity and unitarity: Perturbativity requires all quartic couplings of the scalar potential in Eq. (2.2) obey $\left|\lambda_{i}\right| \leq 8 \pi$. Tree-level unitarity can also be imposed by considering a variety of scattering processes: scalar-scalar scattering, gauge boson-gauge boson scattering and scalargauge boson scattering. We impose these unitarity constraints as derived in [32].

- Vacuum stability: To order to maintain the scalar potential $V$ bounded from below, the following constraints on the IHDM parameters must be met [33]:

$$
\begin{aligned}
& \lambda_{1,2}>0, \quad \lambda_{3}+\lambda_{4}-\left|\lambda_{5}\right|+2 \sqrt{\lambda_{1} \lambda_{2}}>0 \\
& \text { and } \lambda_{3}+2 \sqrt{\lambda_{1} \lambda_{2}}>0
\end{aligned}
$$

- Experimental constraints: For the experimental constraints from electroweak precision tests and collider Higgs searches, we will follow the strategy used in $[32,34,35]$. These constraints can be summarized as follows: (1) $m_{H^{ \pm}}>80 \mathrm{GeV}$ (adapted from chargino search at LEP-II), (2) $\max \left(m_{A}, m_{H^{ \pm}}\right)>100 \mathrm{GeV}$ (adapted from neutralinos search at LEP-II), as well as (3) $m_{A}+m_{S}>m_{Z}$ from the $Z$ width.

\section{Correlation of the signal strengths for $h \rightarrow \gamma \gamma$ and $h \rightarrow Z \gamma$}

Recently, many theoretical works have been devoted to the correlation of the signal strengths between $h \rightarrow \gamma \gamma$ and $h \rightarrow Z \gamma$ in various models, in particular the triplet Higgs models [36-39] and two Higgs doublet models [40,41]. In this section, we will study this correlation in IHDM. In the two processes, one has the same set of charged particles circulating the corresponding loop amplitudes. Feynman diagrams contributing to both $h \rightarrow \gamma \gamma$ and $h \rightarrow Z \gamma$ are depicted in Fig. 1, where all particles inside the loops are shown, with $t, W^{ \pm}, G^{ \pm}, u^{ \pm}$, and $H^{ \pm}$the top quark, the charged gauge bosons, the Goldstone bosons, the Fadeev-Popov ghosts and the charged Higgs bosons respectively. Note that in the case of $h \rightarrow Z \gamma$ the $\gamma-Z$ mixing as depicted generically in the last two diagrams (labeled 29 and 30) of Fig. 1 has to be taken into account. Only after inclusion of such mixing that the amplitude for $h \rightarrow Z \gamma$ becomes ultraviolet finite. Although the final state kinematics as well as the $\gamma$ and $Z$ couplings to the charged particles are different, these two channels should be correlated to certain extent. Compared with SM, the loop amplitudes for the two processes receive an additional contribution from the charged Higgs boson resided in the inert doublet. The partial decay width of $h \rightarrow \gamma \gamma$ can be found in [32], while the one for $h \rightarrow Z \gamma$ it is given by

$$
\begin{aligned}
& \Gamma(h \rightarrow Z \gamma)=\frac{G_{F}^{2} m_{W}^{2} s_{W}^{2} \alpha m_{h}^{3}}{64 \pi^{4}}\left(1-\frac{m_{Z}^{2}}{m_{h}^{2}}\right)^{3} \mid \\
& -2 \frac{\left(3-8 s_{W}^{2}\right)}{3 s_{W} c_{W}}\left(I_{1}\left(\tau_{t}, \lambda_{t}\right)-I_{2}\left(\tau_{t}, \lambda_{t}\right)\right) \\
& \quad-\frac{c_{W}}{s_{W}}\left[4\left(3-\frac{s_{W}^{2}}{c_{W}^{2}}\right) I_{2}\left(\tau_{W}, \lambda_{W}\right)\right. \\
& \left.+\left(\left(1+\frac{2}{c_{W}}\right) \frac{s_{W}^{2}}{c_{W}^{2}}-\left(5+\frac{2}{c_{W}}\right)\right) I_{1}\left(\tau_{W}, \lambda_{W}\right)\right] \\
& +\left.\frac{\left(1-2 s_{W}^{2}\right)}{s_{W} c_{W}} \frac{\left(m_{H^{ \pm}}^{2}-\mu_{2}^{2}\right)}{m_{H^{ \pm}}^{2}} I_{1}\left(\tau_{H^{ \pm}}, \lambda_{H^{ \pm}}\right)\right|^{2}
\end{aligned}
$$

where $\tau_{i}=4 m_{i}^{2} / m_{h}^{2}$ and $\lambda_{i}=4 m_{i}^{2} / m_{Z}^{2}\left(i=t, W^{ \pm}, H^{ \pm}\right)$. We only show the most dominant top quark contribution in the fermion loops. The loop functions $I_{1}$ and $I_{2}$ can be found 

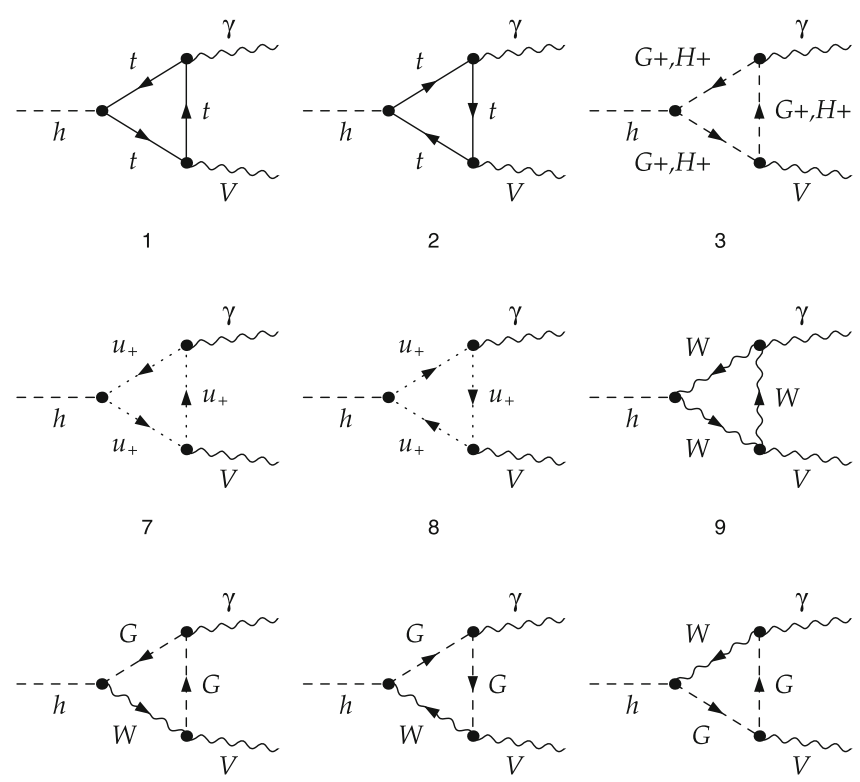

8

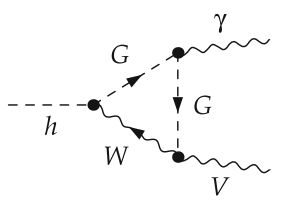

14

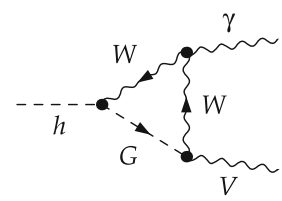

19

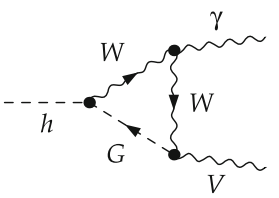

20
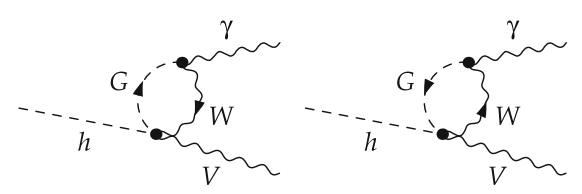

25

26

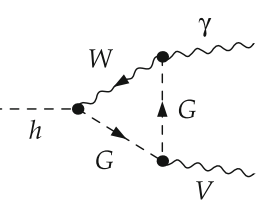

15

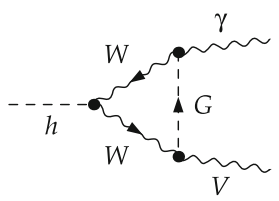

21

27

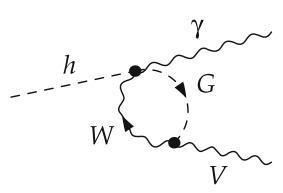

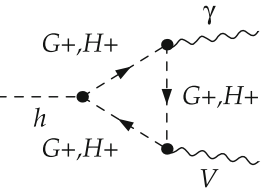

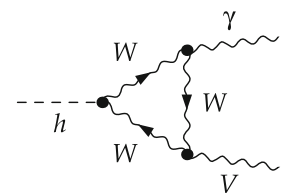

10

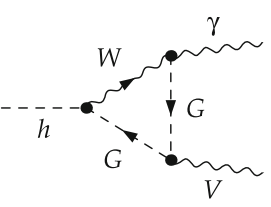

16

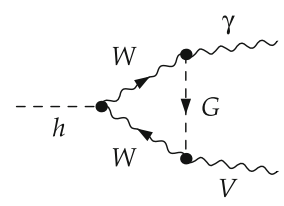

22

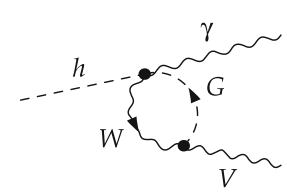

28
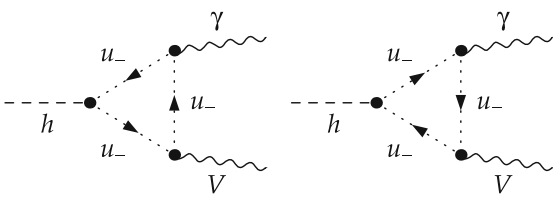

5
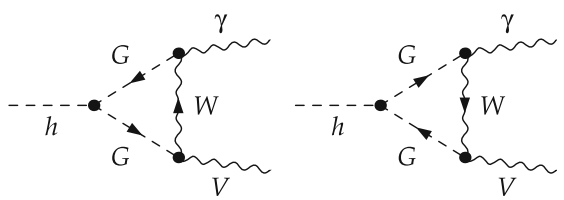

11

12
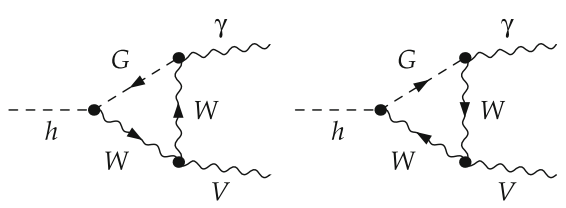

17

18
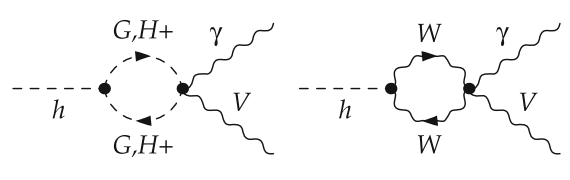

23

24
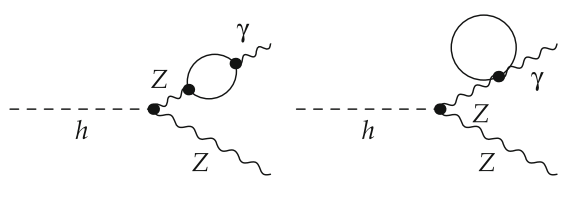

29

Fig. 1 Feynman diagrams for $h \rightarrow \gamma V, V=\gamma$ or $Z$ in the Feynman gauge. Here $t, W^{ \pm}, G^{ \pm}, u^{ \pm}$, and $H^{ \pm}$denote, respectively, the top quark, the charged gauge bosons, the Goldstone bosons, the Fadeev-Popov ghost and the charged Higgs

in the literature [42]. Recall that in the SM, the decay widths of the two processes are dominated by the $W$ loop, which interferes destructively with the subdominant top quark contribution. The extra charged Higgs contribution is shown in the last term of Eq. (3.1), which is proportional to the SM Higgs coupling to a pair of $H^{ \pm}$,

$g_{h H^{ \pm} H^{\mp}}=-2 \frac{m_{W} s_{W}}{e} \lambda_{3}=\frac{e\left(m_{H^{ \pm}}^{2}-\mu_{2}^{2}\right)}{2 m_{W} s_{W}}$

It is clear from Eq. (3.2) that the coupling $g_{h H^{ \pm} H^{\mp}}$ is completely fixed by the parameter $\lambda_{3}$. Just like the case of $h \rightarrow \gamma \gamma$ [32], for negative and positive $\lambda_{3}$, charged Higgs contribution can enhance and suppress the $h \rightarrow Z \gamma$ rate, respectively. A preliminary result for such correlation between $h \rightarrow \gamma \gamma$ and $h \rightarrow Z \gamma$ in the IHDM was first pre- sented $^{1}$ in [43]. Recently this correlation has been discussed in $[34,35,44]$ and similar results were found.

The largest contribution to the production cross section of the Higgs is through gluon fusion. For the Higgs decays to $\gamma \gamma$ or $\gamma Z$ channels, one defines the signal strength as the ratio of production cross section times branching ratio normalized to the $\mathrm{SM}$ one as

$$
\begin{aligned}
R_{\gamma V} & =\frac{\sigma(g g \rightarrow V \gamma)}{\sigma(g g \rightarrow V \gamma)^{S M}} \\
& \approx \frac{\sigma(g g \rightarrow h) \times B r(h \rightarrow V \gamma)}{\sigma(g g \rightarrow h)^{S M} \times B r(h \rightarrow V \gamma)^{S M}}, \quad V=(\gamma, Z)
\end{aligned}
$$

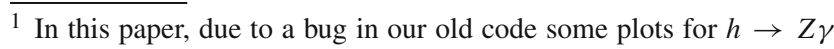
and its correlation with $h \rightarrow \gamma \gamma$ are slightly modified as compared to one presented in [43]. The results now are in agreement with $[34,35]$. 

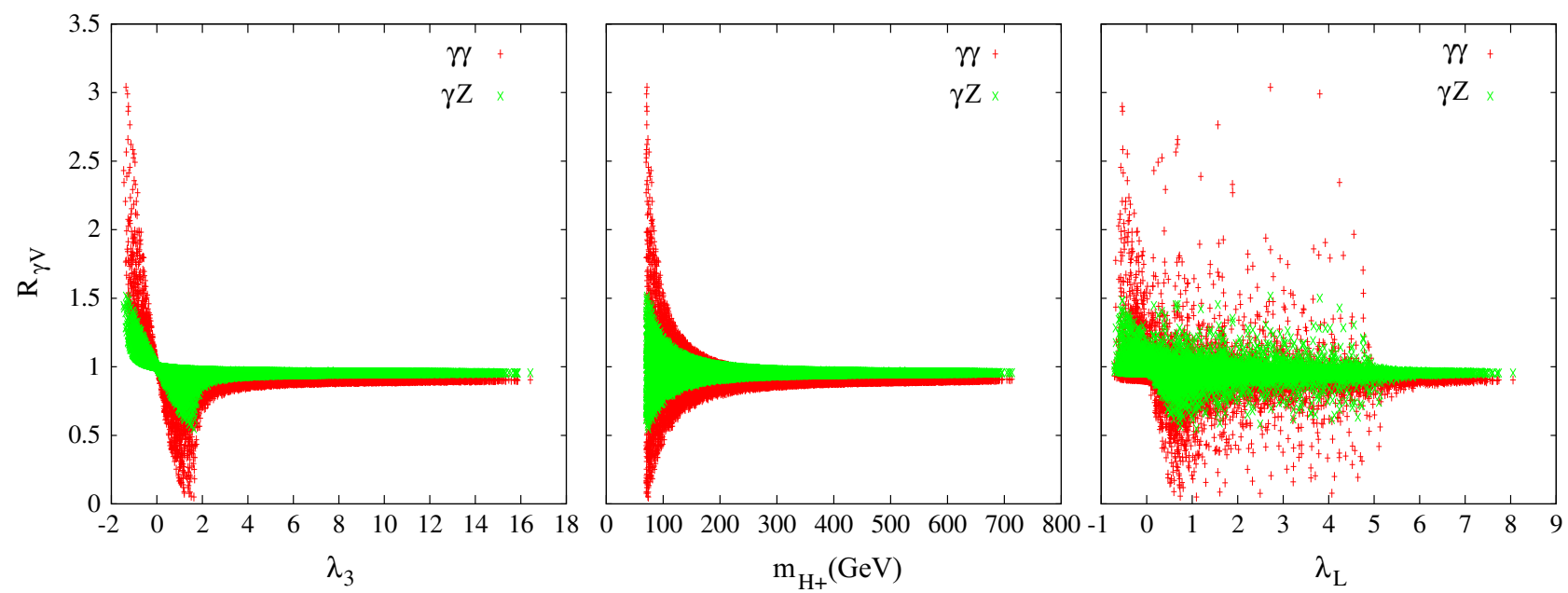

Fig. 2 Signal strength $R_{\gamma V}$ as a function of $\lambda_{3}$ (left), $m_{H^{ \pm}}$(middle) and $\lambda_{L}$ (right). We scan over $m_{H^{ \pm}} \in[70,500] \mathrm{GeV},-10^{6} \leq \mu_{2}^{2} \leq 10^{6} \mathrm{GeV}^{2}$ and $0 \leq \lambda_{2} \leq 4 \pi / 3$

where the narrow width approximation has been used. Since the Higgs $h$ has the same couplings to fermions in IHDM as in $\mathrm{SM}$, the corresponding production cross sections from gluon fusion are the same and Eq. (3.3) reduces to just the ratio of branching ratios. Moreover, if the invisible decay $h \rightarrow \chi \chi$ is not open, the total widths of the Higgs in both models will be approximately the same and Eq. (3.3) will further reduce to the ratio of the partial widths of $h \rightarrow V \gamma$ in both models. In our numerical work, we perform a systematic scan over all the allowed parameter space $\mathcal{P}$ with $m_{h}$ set at $125 \mathrm{GeV}$, taking into account all the theoretical and experimental constraints described in previous section. We note that once the invisible decay $h \rightarrow \chi \chi$ is open, its branching ratio will dominate over all other SM channels unless one tunes the coupling $g_{h \chi \chi}=$ $-2\left(m_{\chi}^{2}-\mu_{2}^{2}\right) / v$ to be very small by taking $m_{\chi}^{2} \approx \mu_{2}^{2}$. The opening of the invisible mode of $h$ would enhance the total width of the SM Higgs boson and therefore suppress both $h \rightarrow \gamma \gamma$ and $h \rightarrow Z \gamma$ branching ratios. We will consider the case where $m_{h}<2 m_{\chi}$ such that the invisible mode of $h \rightarrow \chi \chi$ is close and Eq. (3.3) reduces to just ratio of partial widths as mentioned above.

Results of our scans are depicted in Figs. 2 and 3. In Fig. 2, we plot $R_{\gamma V}$ as a function of $\lambda_{3}$ (left), $m_{H^{ \pm}}$(middle) and $\lambda_{L}$ (right). From the first and second plots, it is clear that to enhance substantially $R_{\gamma \gamma}$ and $R_{\gamma Z}$ we need a negative $\lambda_{3}$ and a rather light charged Higgs. The enhancement in $h \rightarrow$ $Z \gamma$ is always smaller than in $h \rightarrow \gamma \gamma$ because the coupling ratio $g_{Z H^{ \pm} H^{\mp}} / g_{\gamma H^{ \pm} H^{\mp}}=\left(1-2 s_{W}^{2}\right) /\left(2 s_{W} c_{W}\right) \approx 0.67$. The suppression factor of $R_{\gamma Z}$ versus $R_{\gamma \gamma}$ is therefore $(0.67)^{2}$. The lighter the charged Higgs is, the more pronounced in the enhancement of the $\gamma \gamma$ and $Z \gamma$ rates. For instance, if we need $R_{\gamma \gamma} \geq 1.1$ or $R_{\gamma Z} \geq 1.1$ for $\lambda_{3}<0$ (i.e. $\mu_{2}^{2}>m_{H^{ \pm}}^{2}$ ), the charged Higgs mass $m_{H^{ \pm}}$has to be lighter than 200 or 115 $\mathrm{GeV}$, respectively. In the rightmost of Fig. 2, we plot $R_{\gamma V}$ as a function of $\lambda_{L}$ in the range of $[-2,2]$. We note that $\lambda_{L}$ is

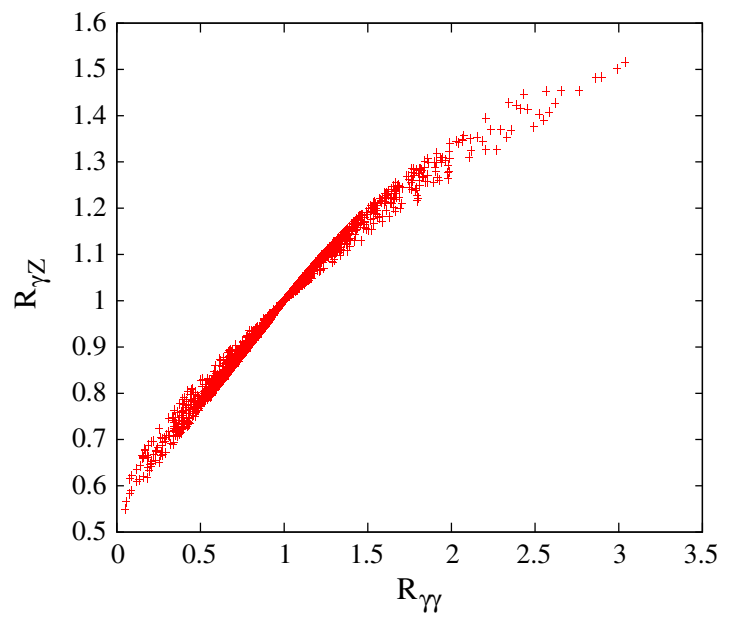

Fig. 3 Correlation between $R_{\gamma \gamma}$ and $R_{\gamma Z}$ in IHDM. Parameter scan same as Fig. 2

an important parameter which enters in the calculation of the relic density of DM and hence it is constrained by the WMAP data to be in the range of $\left|\lambda_{L}\right|<0.2$ [45]. More sophisticated limits of this parameter $\lambda_{L}$ depending on the mass $m_{\chi}$ have been deduced recently from a global analysis of IHDM [30]. We note that the stringent limits obtained in [30] all lie within the range of $[-2,2]$, thus some enhancements in $R_{\gamma \gamma}$ and $R_{\gamma Z}$ are still possible according to this plot. The correlation between $R_{\gamma \gamma}$ and $R_{\gamma Z}$ is roughly a linear one as shown in Fig. 3 using the same parameter scan as in Fig. 2. From the plot, one finds that for $R_{\gamma \gamma}>1$ where the $W^{ \pm}$and $H^{ \pm}$ loops interfere constructively, we have $R_{\gamma \gamma} \geq R_{\gamma Z}$; while in the opposite case of $R_{\gamma \gamma}<1$ where the $W^{ \pm}$and $H^{ \pm}$ loops interfere destructively, we can have $R_{\gamma \gamma} \leq R_{\gamma Z}$. The main reason for this feature is that the destructive interference between $W^{ \pm}$and $H^{ \pm}$is more effective in $R_{\gamma \gamma}$ than in $R_{\gamma Z}$ since the latter process has a much larger $W^{ \pm}$contribution. 

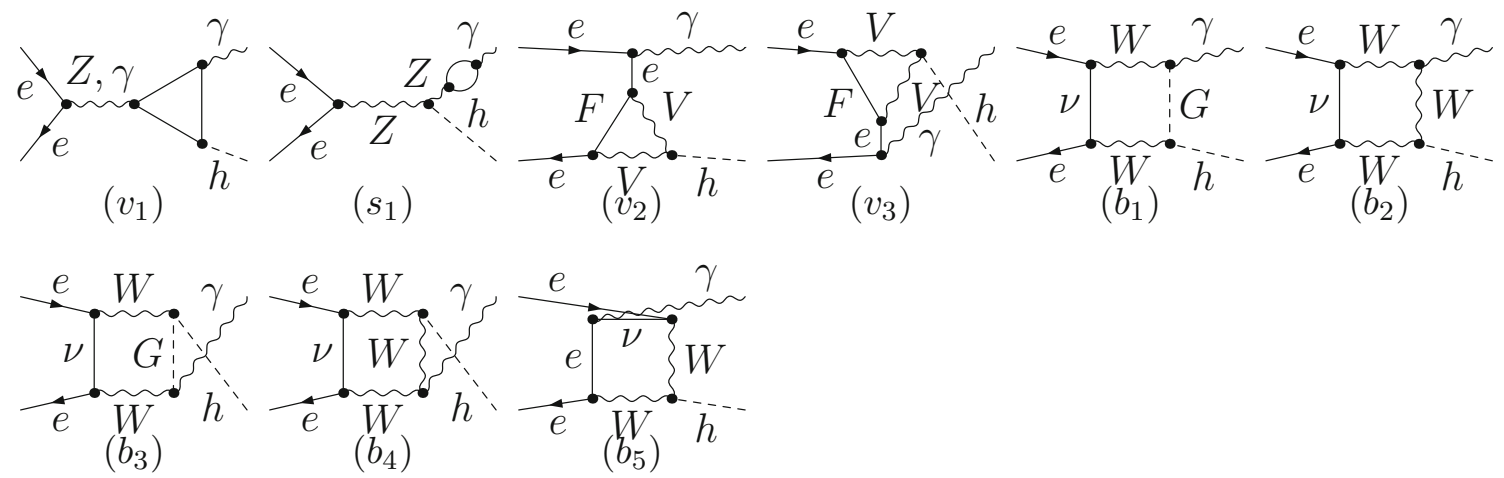

Fig. 4 Generic Feynman diagrams contributing to $e^{-} e^{+} \rightarrow h \gamma$. For the particles inside the loops in diagrams $v_{1}$, we have all possible charged particles given in Fig. 1 . The mixing $\gamma-Z$ diagram $s_{1}$ receives contributions from all SM particles as well as charged Higgs. For diagrams $v_{2,3}, V$ denotes $Z$ or $W$, while $F$ denotes $e$ or $v$. Box diagrams $b_{1, \ldots, 5}$ are necessary for gauge invariance
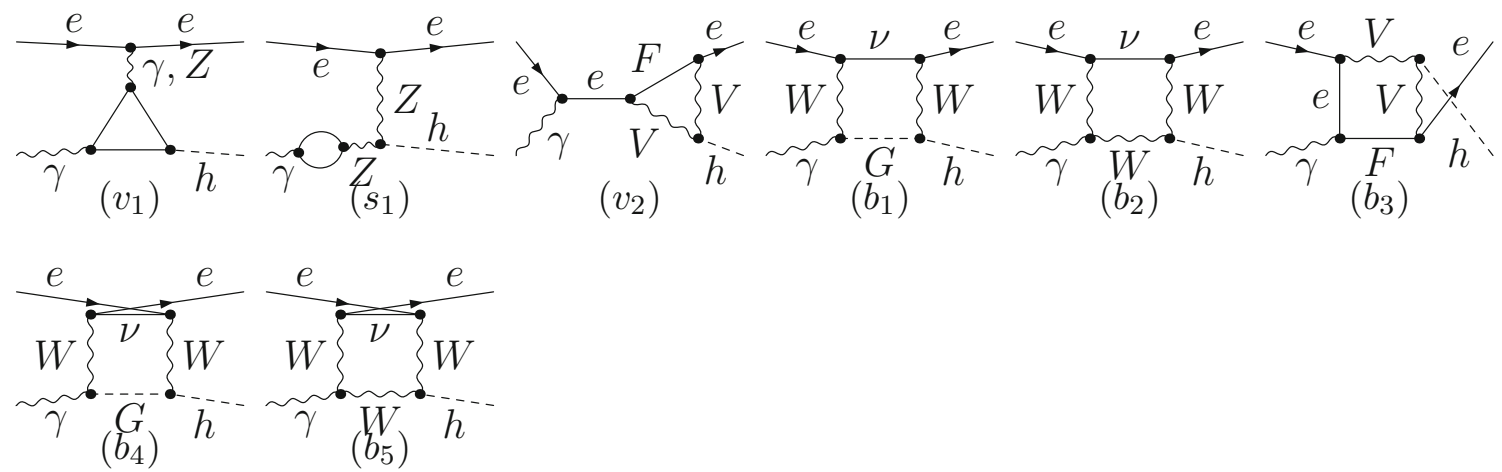

Fig. 5 Generic Feynman diagrams contributing to $e^{-} \gamma \rightarrow e^{-} h$. For the particles inside the loops in diagrams $v_{1}$, we have all possible charged particles like in Fig. 1 . The mixing $\gamma-Z$ diagram $s_{1}$ receives

\section{Associated production $e^{+} e^{-} \rightarrow \gamma h$ and $e^{-} \gamma \rightarrow e^{-} h$ in IHDM}

At tree level, the associated production process of $e^{+} e^{-} \rightarrow$ $\gamma h$ is mediated by t-channel electron exchange diagram which is suppressed by the electron mass. For the process $e^{-} \gamma \rightarrow e^{-} h$, the tree-level contribution is mediated by schannel diagrams which is also suppressed by the electron mass. At one-loop level, they are mediated by triangle, selfenergy as well as box diagrams and hence they are sensitive to all virtual particles (physical gauge bosons, fermions and charged Higgs particles as well as unphysical Goldstone $G^{ \pm}$and ghost particles $u^{ \pm}$) inside the loop. We display in Figs. 4 and 5 some generic Feynman diagrams that contribute to $e^{+} e^{-} \rightarrow \gamma h$ and $e^{-} \gamma \rightarrow e^{-} h$ respectively, indicating that some individual amplitudes are sensitive to the off-shell $h \gamma V^{*}$ vertices. Both in Figs. 4 and 5, diagrams $v_{1}$ are generic one and the particles content is depicted in Fig. 1. The process $e^{+} e^{-} \rightarrow \gamma h$ had been studied in SM long time ago $[46,47]$. Effects from new physics to this process had been contributions from all SM particles as well as charged Higgs. For diagram $v_{2}, V$ denotes $Z$ or $W$, while $F$ denotes $e$ or $v$. Box diagrams $b_{1, \ldots, 5}$ are necessary for gauge invariance

analyzed in Ref. [48] for supersymmetry and Ref. [49,50] for an extended Higgs sector.

Our calculation is done in Feynman gauge using dimensional regularization with the help of FeynArts and FormCalc packages [51-54]. Numerical evaluation of the scalar integrals is done with LoopTools $[55,56]$. Throughout the calculation we will neglect the electron mass. Since the tree-level amplitudes which are suppressed by the electron mass are neglected, Feynman diagrams like Fig. 4- $v_{2}, v_{3}$ and Fig. 5- $v_{2}$ are ultraviolet finite because the corresponding counter-terms for $e^{+} e^{-} h$ are proportional to electron mass. We have checked both analytically and numerically that the total amplitudes for the two processes are ultraviolet finite. The $\gamma-Z$ self-energy mixing is necessary in order to achieve the finite results. While the fermionic contributions to $v_{1}$ (triangle) and $s_{1}$ (self-energy) diagrams in Figs. 4 and 5 are gauge invariance by themselves, for gauge boson diagrams we need to sum these with all other (triangle and box) diagrams in order to maintain gauge invariance in the final results [48]. In all Feynman diagrams computed here, there 


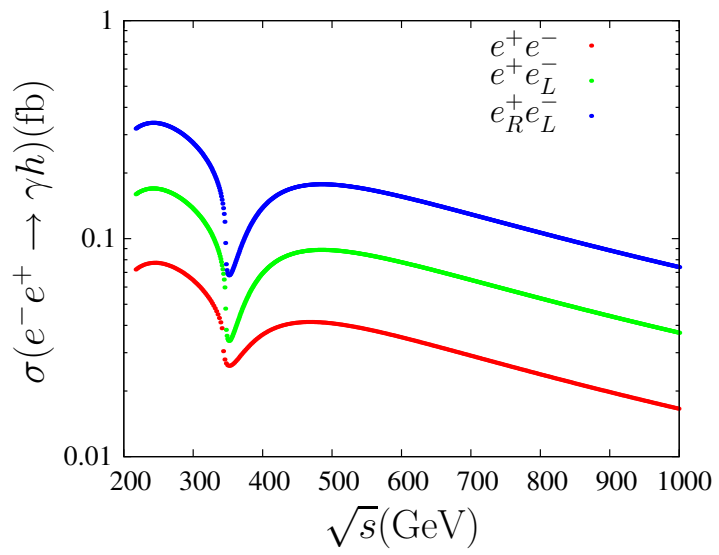

Fig. 6 Total cross section for $e^{+} e^{-} \rightarrow \gamma h$ (fb) in the SM as a function of center-of-mass energy with $m_{h}=125 \mathrm{GeV}$. From bottom to top: unpolarized, left polarized electron, left polarized electron and right polarized positron

is no virtual photon in the loops, therefore the results are infrared finite. Real or virtual emission of the photon is suppressed by the electron mass. For illustrative purpose in the following, it is convenient to introduce the two ratios

$R_{\gamma h} \equiv \frac{\sigma\left(e^{+} e^{-} \rightarrow \gamma h\right)}{\sigma_{\mathrm{SM}}\left(e^{+} e^{-} \rightarrow \gamma h\right)}, \quad R_{e^{-} h} \equiv \frac{\sigma\left(e^{-} \gamma \rightarrow e^{-} h\right)}{\sigma_{\mathrm{SM}}\left(e^{-} \gamma \rightarrow e^{-} h\right)}$,

which are the total cross sections in the IHDM normalized to the SM one.

\section{$4.1 e^{+} e^{-} \rightarrow \gamma h$}

In Fig. 6, we plot the associated production cross section of the $125 \mathrm{GeV}$ SM Higgs with a photon at the linear collider as a function of center-of-mass energy $\sqrt{s}$ from $200 \mathrm{GeV}$ to $1 \mathrm{TeV}$. The lower, middle and upper curves correspond to the unpolarized $e^{+} e^{-}$, polarized $e^{+} e_{L}^{-}$and $e_{R}^{+} e_{L}^{-}$beams respectively. In all three cases, the cross sections are enhanced near the region of $\sqrt{s} \approx 250 \mathrm{GeV}$. As the center-of-mass energy increases further, the destructive interference between the top quark and $W^{ \pm}$contributions get more severe and become maximal near the $t \bar{t}$ threshold, responsible for the dips clearly seen in the figure. After crossing the $t \bar{t}$ threshold, the cross sections scale like $1 / s$ and thus drop steeply. Note that with the polarization of the initial state of positron or both electron and positron the total cross section can be increased by roughly a factor 2 or 4 , respectively, compared with the unpolarized case. In Fig. 7 we exhibit the corresponding angular distribution $d \sigma / d \cos \theta$ at $\sqrt{s}=250$ (left) and 500 (right) $\mathrm{GeV}$, respectively. One observes that at both energies the distributions are rather symmetric for either choices of polarized or unpolarized beams.

In Fig. 8 we show the total cross section $e^{+} e^{-} \rightarrow \gamma h$ as a function of $\lambda_{3}$ (left) and the correlation between $R_{\gamma \gamma}$ and $R_{\gamma h}$ (right) for $\lambda_{2}=3.75$ and $m_{A}=m_{S}=m_{H^{ \pm}}+10 \mathrm{GeV}$ with $m_{H^{ \pm}} \in[90,350] \mathrm{GeV}$. Upper and lower plots are for $\sqrt{s}=250$ and $500 \mathrm{GeV}$ respectively.

For $\sqrt{s}=250 \mathrm{GeV}$ (two upper plots in Fig. 8), it is clear that when $\lambda_{3}$ is negative, interference of $H^{ \pm}$and $W^{ \pm}$loops in the off-shell $h \gamma V^{*}(V=\gamma, Z)$ amplitudes is also constructive and it can give rise to some enhancement in the total cross section of $e^{+} e^{-} \rightarrow \gamma h$ with respect to SM. The increase can be as large as a factor of 2. This large enhancement requires of course a rather light charged Higgs in the range $[90,200] \mathrm{GeV}$ circulating inside the loop. Given the fact that the enhancement of the cross section happens for negative $\lambda_{3}$, which is the same condition as having an enhancement in $R_{\gamma \gamma}$ and $R_{\gamma Z}$, the correlation between $R_{\gamma \gamma}$ and $R_{\gamma h}$ is shown in the upper right plot of Fig. 8. Clearly, when $R_{\gamma \gamma}>1$ we also have $R_{\gamma h}>1$. Similar correlation can be found between $R_{\gamma Z}$ and $R_{\gamma h}$ but will not be shown here.

For $\sqrt{s}=500 \mathrm{GeV}$ (two lower plots in Fig. 8), the top quark contribution gets amplified after crossing the $t \bar{t}$ threshold which leads to more destructive interference with the $W^{ \pm}$ loops as can be seen in the lower left plot in Fig. 8 for both positive and negative $\lambda_{3}$. At this higher energy, the destructive
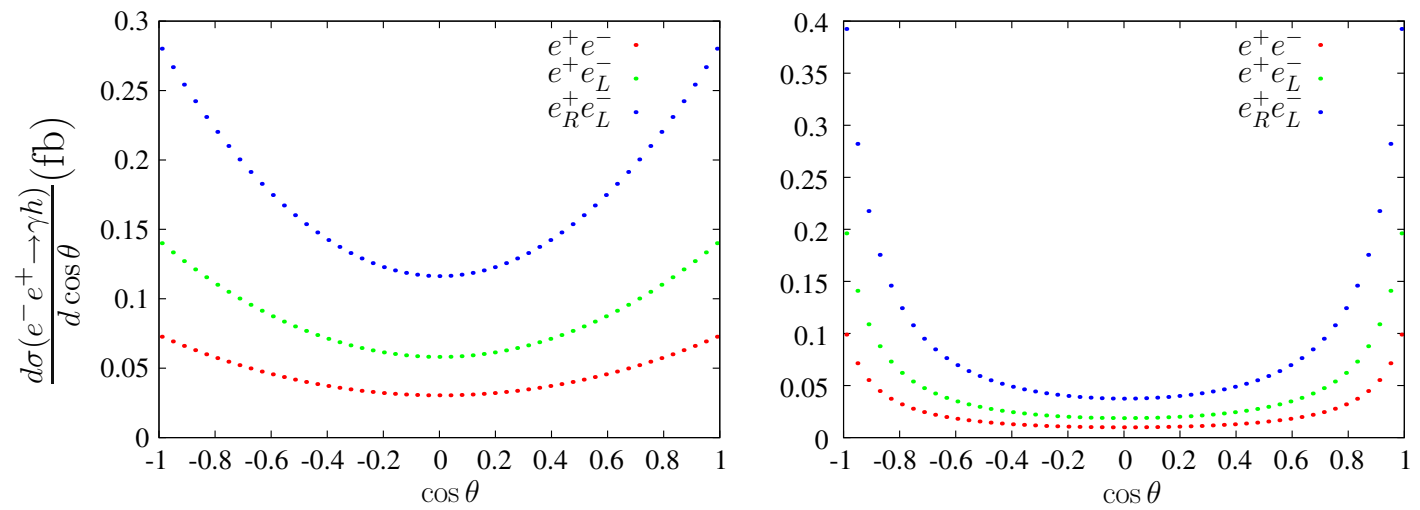

Fig. 7 Differential cross section for $e^{+} e^{-} \rightarrow \gamma h(\mathrm{fb})$ in the SM with $m_{h}=125 \mathrm{GeV}$ and $\sqrt{s}=250($ left $)$ and $500($ right $) \mathrm{GeV}$ 

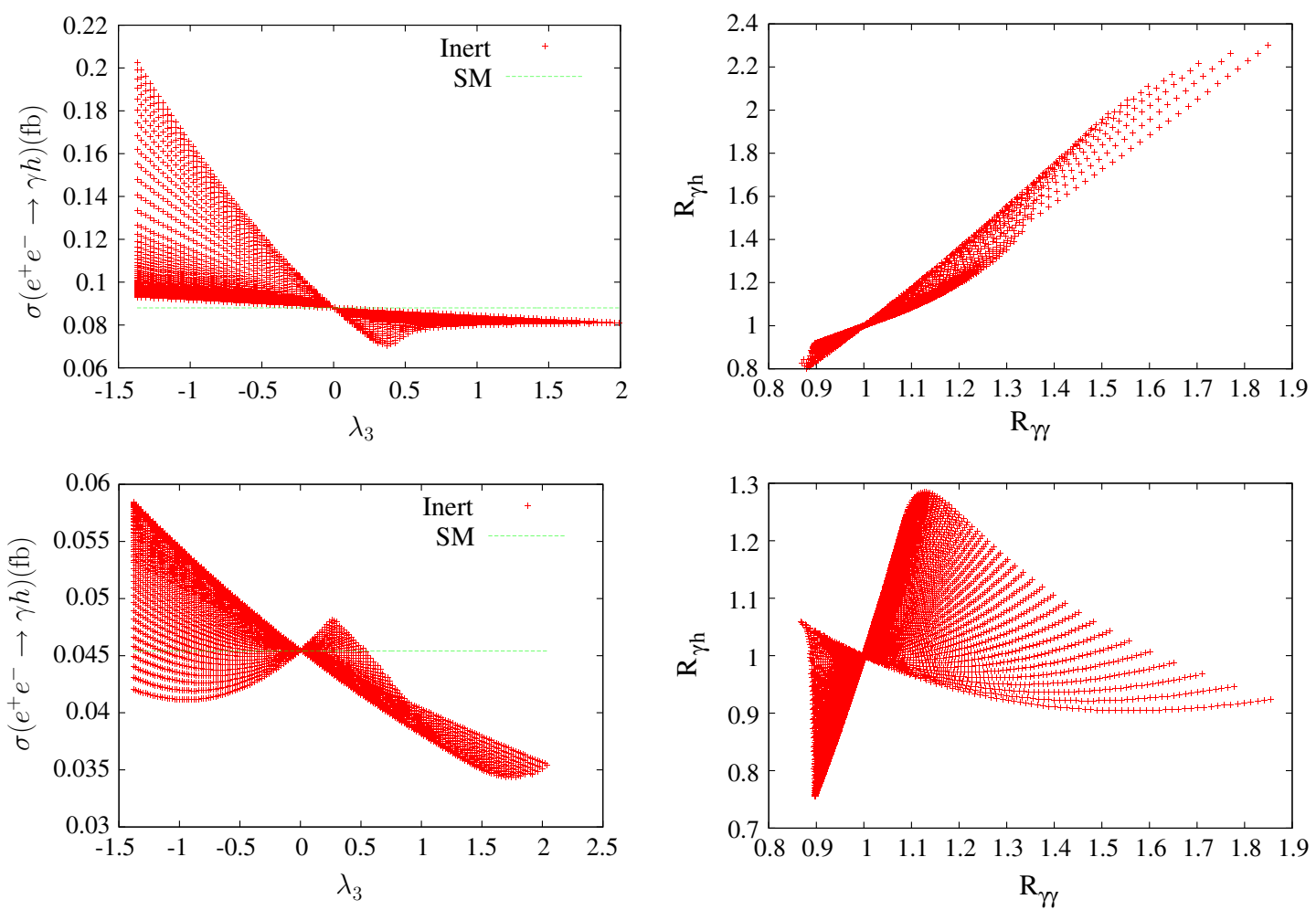

Fig. 8 Total cross section for $e^{+} e^{-} \rightarrow \gamma h$ in IHDM as a function of $\lambda_{3}$ (left) and correlation between $R_{\gamma \gamma}$ and $R_{\gamma h}$ (right). Input parameters are $m_{h}=125 \mathrm{GeV}, \lambda_{2}=3.75, \mu_{2}^{2} \in[0,5] \mathrm{TeV}^{2}$, and

$m_{A}=m_{S}=m_{H^{ \pm}}+10 \mathrm{GeV}$ with $m_{H^{ \pm}} \in[90,350] \mathrm{GeV}$. Upper and lower plots correspond to $\sqrt{s}=250$ and $500 \mathrm{GeV}$ respectively

top quark loop can overwhelm the constructive interference between the $H^{ \pm}$and $W^{ \pm}$loops with negative $\lambda_{3}$ such that the total cross section is below its SM value. The opposite case is also possible; the top quark loop can be in constructive interference with the $H^{ \pm}$loop for positive $\lambda_{3}$ and overwhelm the $W$ loop leading to a total cross section larger than its SM value. In the lower right plot of Fig. 8, the correlation between $R_{\gamma \gamma}$ and $R_{\gamma h}$ is also shown for $\sqrt{s}=500 \mathrm{GeV}$.

\section{$4.2 e^{-} \gamma \rightarrow e^{-} h$}

Next we turn to a discussion of the case of $e^{-} \gamma \rightarrow e^{-} h$. If the $e^{-} \gamma$ option for ILC is made available, the center-of-mass energy may be slightly reduced as compared with the previous $e^{+} e^{-}$case shown in Fig. 7. In the left plot in Fig. 9, we show the differential cross section $d \sigma\left(e^{-} \gamma \rightarrow e^{-} h\right) / d \cos \theta$ for three center-of-mass energies at $\sqrt{s}=200,280$ and $400 \mathrm{GeV}$. Obviously, the differential cross section for this $e^{-} \gamma$ case get significantly enhanced near the forward direction $\cos \theta \approx 1$ due to the $t$ channel singularity between the incoming and outgoing electrons. To avoid this kinematical singularity we will impose a cut on the scattering angle when computing the total cross section. In the right plot in Fig. 9, we illustrate the total cross section $\sigma\left(e^{-} \gamma \rightarrow e^{-} h\right)$ as a function of center-of-mass energy for three different cuts of $10^{\circ}, 20^{\circ}$ and $30^{\circ}$ on the scattering angle. The sensitivity to

the angular cut is quite evident. At $\sqrt{s} \approx 250 \mathrm{GeV}$, the total cross section can reach a maximum value of $1.38,0.95$ and $0.8 \mathrm{fb}$ for $10^{\circ}, 20^{\circ}$ and $30^{\circ}$ cuts, respectively. We will use a $20^{\circ}$ cut on the scattering angle in our next figure.

In the left plot of Fig. 10 we show the total cross section for $e^{-} \gamma \rightarrow e^{-} h$ as a function of $\lambda_{3}$ for $\sqrt{s}=200$ and $400 \mathrm{GeV}$. It is clear that the charged Higgs loop interferes constructively (destructive) with the SM loops for negative (positive) $\lambda_{3}$, respectively. The lighter the charged Higgs mass is, the larger the enhancement in the total cross section $\sigma\left(e^{-} \gamma \rightarrow e^{-} h\right)$. In the right plot of Fig. 10, the correlation between $R_{\gamma \gamma}$ and $R_{e^{-} h}$ is shown for $\sqrt{s}=200$ and $400 \mathrm{GeV}$. At both energies, we have a positive correlation and $R_{\gamma \gamma} \approx R_{e^{-} h}$. A similar conclusion can be drawn for the correlation between $R_{\gamma Z}$ and $R_{e^{-} h}$, which we will not show here.

\section{Conclusions}

Despite the discovery of the SM Higgs, the search for physics beyond the SM is just getting started. Since all the current LHC and Tevatron data point toward this new boson is indeed the SM Higgs with its couplings consistent with the SM expectations, it leaves little room for an extra Higgs doublet to play any role in the spontaneous symmetry breaking. 

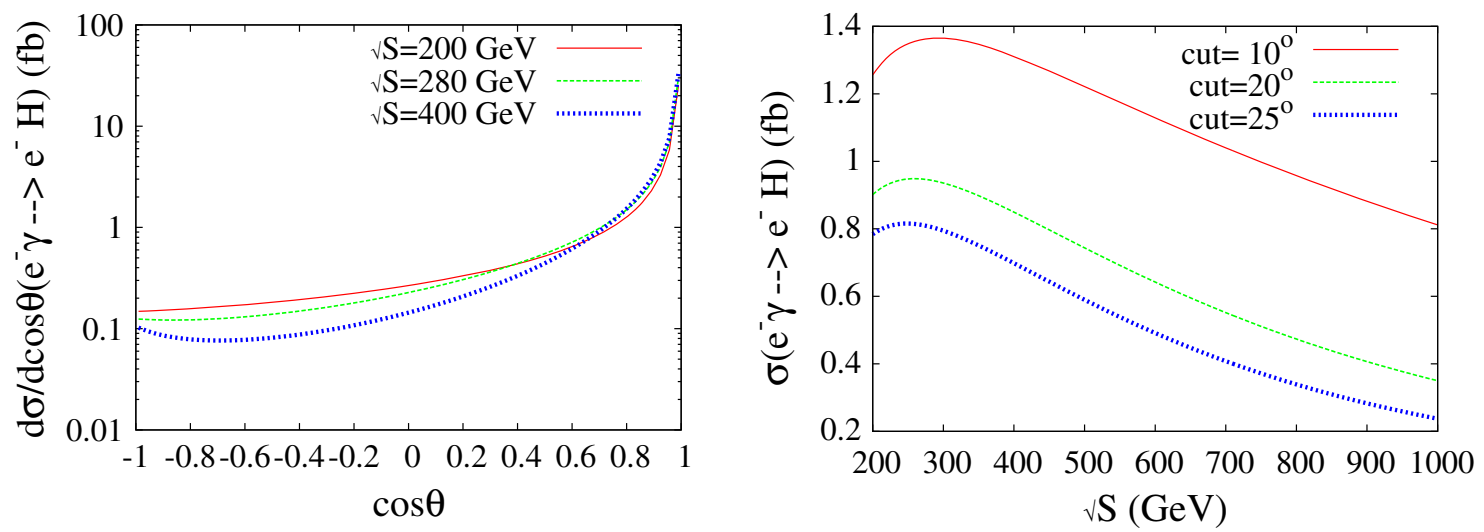

Fig. 9 (Left) SM differential cross section for $e^{-} \gamma \rightarrow e^{-} h$ with $m_{h}=125 \mathrm{GeV}$ for $\sqrt{s}=200 \mathrm{GeV}$ (red), $280 \mathrm{GeV}$ (green) and $400 \mathrm{GeV}($ blue). (Right) SM total cross section as a function of center-of-mass energy for three different angle cuts of $10^{\circ}$ (red), $20^{\circ}$ (green) and $25^{\circ}$ (blue)
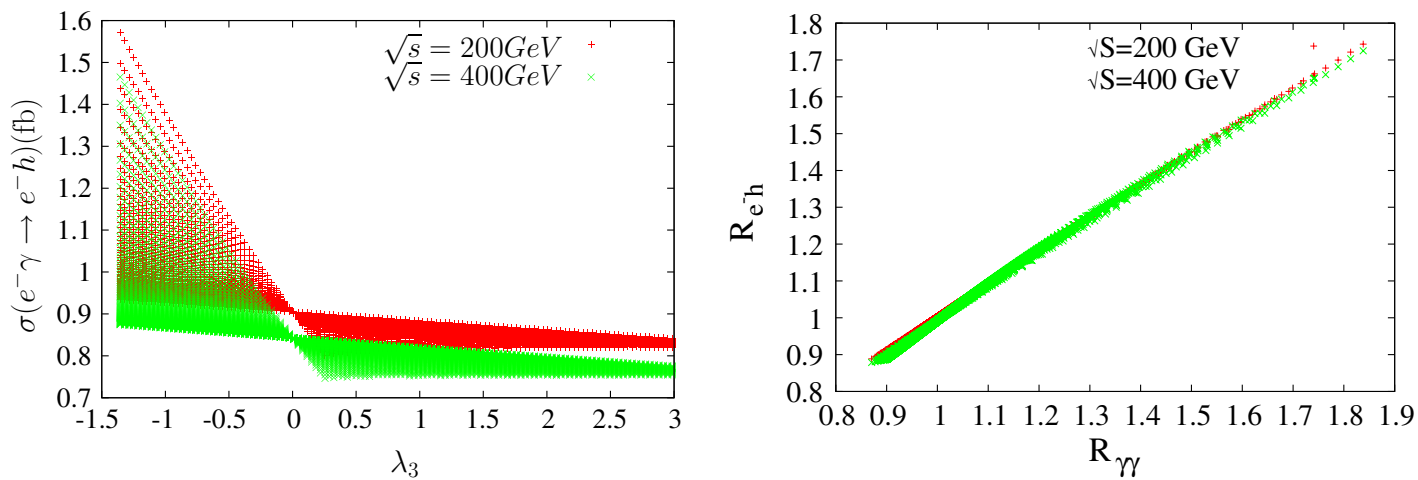

Fig. 10 Total cross section for $e^{-} \gamma \rightarrow e^{-} h$ in IHDM as a function of $\lambda_{3}(l e f t)$ and correlation between $R_{\gamma \gamma}$ and $R_{e^{-} h}(r i g h t)$ for $\sqrt{s}=200 \mathrm{GeV}$ (red) and $400 \mathrm{GeV}$ (green). Parameter scan same as Fig. 8

In this regard, IHDM is quite an interesting model beyond the SM since its extra Higgs doublet is inert.

In Sect. 3 of this paper we analyzed the correlation between the LHC signal strengths $R_{\gamma \gamma}(h)$ and $R_{\gamma Z}(h)$ in the IHDM with the possible deviation from their SM predictions of unity. We have considered the scenario where the invisible modes are not opened. In this case, we have shown that $R_{\gamma \gamma}(h)$ and $R_{\gamma Z}(h)$ are positively correlated with a roughly linear relation between the two. Depending on whether $R_{\gamma \gamma}(h)$ is greater or smaller than 1, we found that $R_{\gamma Z}(h)$ is smaller or greater than $R_{\gamma \gamma}(h)$, respectively, due to the intricate destructive interference between the contribution of the additional charged Higgs with the SM $W^{ \pm}$ inside the loop of the two processes.

While the decay mode $h \rightarrow \gamma \gamma$ has played an important role in the discovery of the SM Higgs at the LHC, the mode $h \rightarrow Z \gamma$ has yet to be verified. We expect that LHC-14 should be able to detect the latter mode positively and provide useful correlation information among these two modes. The correlation of these two signal strengths studied in this work for IHDM can then be tested accordingly at the LHC14.
Due to its clean environment, ILC has great potential to measure various properties of the SM Higgs more precisely. These include branching ratios, cross sections, $\mathrm{CP}$ properties and its mass. In Section IV of this paper, we have computed the one-loop processes $e^{+} e^{-} \rightarrow \gamma h$ and $e^{-} \gamma \rightarrow e^{-} h$ in the Feynman gauge using dimensional regularization for the future ILC machine. We have shown that the charged Higgs loops in IHDM can modify the SM predictions for these processes in a significant way. For both processes, we have calculated in the IHDM the total as well as the differential cross section for the recently discover Higgs at $125 \mathrm{GeV}$. We also studied the total cross sections for these two processes as a function of the parameter $\lambda_{3}$, which controls the contribution of the charged Higgs boson in the loops. We find that the cross sections for both processes are quite sensitive to this parameter so that the signal strengths $R_{\gamma h}$ and $R_{e^{-} h}$ that we defined for the ILC might deviate from their SM values of unity. Furthermore, we have studied the correlation of these two signal strengths with $R_{\gamma \gamma}$. We found that for the correlation between $R_{\gamma h}$ and $R_{\gamma \gamma}$ can be mainly positively for $\sqrt{s}=250 \mathrm{GeV}$ and either positive or negative correlated for $\sqrt{s}=500 \mathrm{GeV}$ depending on the IHDM parameter 
space. On the other hand, for the correlation between $R_{e^{-}} h$ and $R_{\gamma \gamma}$, we found a roughly linear relation between them for both $\sqrt{s}=200$ and $500 \mathrm{GeV}$. All our predictions for the IHDM in this work can be tested at the ILC.

Acknowledgments AA would like to thank the hospitality of Institute of Physics, Academia Sinica in Taiwan where progress of this work was made. This work was supported in part by the Spanish Consejo Superior de Investigaciones Cientificas (CSIC) (RB) and by the National Science Council of Taiwan under project number 102-2811-M-001-032 (AA) and grant number 101-2112-M-001-005-MY3 (TCY).

Open Access This article is distributed under the terms of the Creative Commons Attribution License which permits any use, distribution, and reproduction in any medium, provided the original author(s) and the source are credited.

Funded by $\mathrm{SCOAP}^{3}$ / License Version CC BY 4.0.

\section{References}

1. G. Aad et al. [ATLAS Collaboration], Phys. Lett. B 716, 1 (2012) http://arxiv.org/abs/1207.7214 [hep-ex]

2. S. Chatrchyan et al. [CMS Collaboration], Phys. Lett. B 716, 30 (2012) http://arxiv.org/abs/1207.7235 [hep-ex]

3. Tevatron New Physics Higgs Working Group and CDF and D0 Collaborations. http://arxiv.org/abs/1207.0449 [hep-ex]

4. ATLAS Collaboration, ATLAS-CONF-2013-014 (2013). https:// cds.cern.ch/record/1523727

5. CMS Collaboration, CMS-PAS-HIG-13-005 (2013). https://cds. cern.ch/record/1542387

6. G. Aad et al. [ATLAS Collaboration], Phys. Lett. B 726, 88 (2013) http://arxiv.org/abs/1307.1427 [hep-ex]

7. ATLAS Collaboration, ATLAS-CONF-2013-034 (2013). https:// cds.cern.ch/record/1528170

8. S. Chatrchyan et al. [CMS Collaboration], JHEP 06, 081 (2013). http://arxiv.org/abs/1303.4571 [hep-ex]

9. F. Englert, R. Brout, Phys. Rev. Lett. 13, 321 (1964)

10. P.W. Higgs, Phys. Rev. Lett. 13, 508 (1964)

11. L.D. Landau, Dokl. Akad. Nauk Ser. Fiz. 60, 207 (1948)

12. C.-N. Yang, Phys. Rev. 77, 242 (1950)

13. S. Bolognesi, Y. Gao, A.V. Gritsan, K. Melnikov, M. Schulze, N.V. Tran, A. Whitbeck, Phys. Rev. D 86, 095031 (2012). arXiv:1208.4018 [hep-ph]

14. G. Aad et al. [ATLAS Collaboration], Phys. Lett. B 726, 120 (2013). http://arxiv.org/abs/1307.1432 [hep-ex]

15. G. Aad et al. [ATLAS Collaboration], ATLAS-CONF-2013-040

16. S. Chatrchyan et al. [CMS Collaboration], Phys. Rev. Lett. 110, 081803 (2013). http://arxiv.org/abs/1212.6639 [hep-ex]

17. S. Chatrchyan et al. [CMS Collaboration], CMS-PAS-HIG-13-002

18. Technical Design Report, The ILC Baseline Design, https://forge. linearcollider.org/dist/20121210-CA-TDR2 (2013)

19. H. Baer, T. Barklow, K. Fujii, Y. Gao, A. Hoang, S. Kanemura, J. List, H.E. Logan et al., arXiv:1306.6352 [hep-ph]

20. G. Weiglein et al., LHC/LC Study Group Collaboration. Phys. Rept. 426, 47 (2006). [hep-ph/0410364]

21. Detector Baseline Document, http://ific.uv.es/ fuster/DBDChapters/ (2013)

22. Parameters for the Linear Collider (2003, updated 2006). http://www.fnal.gov/directorate/icfa/recent_lc_activities_files/ para-Nov20-final

23. M.E. Peskin. arXiv:1207.2516 [hep-ph]

24. N.G. Deshpande, E. Ma, Phys. Rev. D 18, 2574 (1978)

25. E. Ma, Phys. Rev. D 73, 077301 (2006). [hep-ph/0601225]
26. R. Barbieri, L.J. Hall, V.S. Rychkov, Phys. Rev. D 74, 015007 (2006). [hep-ph/0603188]

27. M. Gustafsson, E. Lundstrom, L. Bergstrom, J. Edsjo, Phys. Rev. Lett. 99, 041301 (2007). [astro-ph/0703512 [ASTRO-PH]]

28. A. Goudelis, B. Herrmann, O. Stal, JHEP 1309, 106 (2013). arXiv: 1303.3010 [hep-ph]

29. A. Pierce, J. Thaler, JHEP 0708, 026 (2007). [hep-ph/0703056 [HEP-PH]

30. A. Arhrib, Y. -L. S. Tsai, Q. Yuan and T. -C. Yuan, arXiv:1310.0358 [hep-ph]

31. I.F. Ginzburg, K.A. Kanishev, M. Krawczyk, D. Sokolowska, Phys. Rev. D 82, 123533 (2010). arXiv:1009.4593 [hep-ph]

32. A. Arhrib, R. Benbrik, N. Gaur, Phys. Rev. D 85, 095021 (2012). arXiv: 1201.2644 [hep-ph]

33. G.C. Branco, P.M. Ferreira, L. Lavoura, M.N. Rebelo, M. Sher, J.P. Silva, Phys. Rept. 516, 1 (2012). arXiv:1106.0034 [hep-ph]

34. B. Swiezewska, M. Krawczyk, Phys. Rev. D 88, 035019 (2013). arXiv: 1212.4100 [hep-ph]

35. E. Lundstrom, M. Gustafsson, J. Edsjo, Phys. Rev. D 79, 035013 (2009). arXiv:0810.3924 [hep-ph]

36. C.-S. Chen, C.-Q. Geng, D. Huang, L.-H. Tsai, Phys. Lett. B 723, 156 (2013). arXiv:1302.0502 [hep-ph]

37. C.-S. Chen, C.-Q. Geng, D. Huang, L.-H. Tsai, Phys. Rev. D 87, 075019 (2013). arXiv:1301.4694 [hep-ph]

38. P.S. Bhupal Dev, D.K. Ghosh, N. Okada, I. Saha, JHEP 303, 150 (2013) [Erratum-ibid. 1305,049 (2013)]. http://arxiv.org/abs/1301. 3453 [hep-ph]

39. C.-W. Chiang, K. Yagyu, Phys. Rev. D 87, 033003 (2013). arXiv:1207.1065 [hep-ph]

40. G. Bhattacharyya, D. Das, P.B. Pal, M.N. Rebelo, arXiv:1308.4297 [hep-ph]

41. A. Arhrib, W. Hollik, S. Penaranda, M. Capdequi, Peyranere. Phys. Lett. B 579, 361 (2004)

42. J.F. Gunion, H.E. Haber, G.L. Kane, S. Dawson, The Higgs Hunter's Guide. Front. Phys. 80, 1 (2000)

43. Talk given by A. Arhrib at Toyama International Workshop on "Higgs as a Probe of New Physics 2013" (HPNP2013). http://jodo. sci.u-toyama.ac.jp/theory/HPNP2013/

44. M. Krawczyk, D. Sokolowska, P. Swaczyna, B. Swiezewska, arXiv: 1309.7880 [hep-ph]

45. E.M. Dolle, S. Su, Phys. Rev. D 80, 055012 (2009). arXiv:0906.1609 [hep-ph]

46. A. Barroso, J. Pulido, J.C. Romao, Nucl. Phys. B 267, 509 (1986)

47. A. Abbasabadi, D. Bowser-Chao, D.A. Dicus, W.W. Repko, Phys. Rev. D 52, 3919 (1995). [hep-ph/9507463]

48. A. Djouadi, V. Driesen, W. Hollik, J. Rosiek, Nucl. Phys. B 491, 68 (1997). [hep-ph/9609420]

49. M. Krawczyk, J. Zochowski, P. Mattig, Eur. Phys. J. C 8, 495 (1999). [hep-ph/9811256]

50. A.G. Akeroyd, A. Arhrib, M. Capdequi Peyranère, Mod. Phys. Lett. A 14, 2093 (1999) [Erratum-ibid. A 17, 373 (2002)] [hep-ph/9907542]

51. T. Hahn, Comput. Phys. Commun. 140, 418 (2001)

52. T. Hahn, C. Schappacher, Comput. Phys. Commun. 143, 54 (2002)

53. T. Hahn, M. Perez-Victoria, Comput. Phys. Commun. 118, 153 (1999)

54. J. Küblbeck, M. Böhm, A. Denner, Comput. Phys. Commun. 60, 165 (1990)

55. G.J. van Oldenborgh, Comput. Phys. Commun. 66, 1 (1991)

56. T. Hahn, Acta Phys. Polon. B 30, 3469 (1999), PoS ACAT 2010, 078 (2010) arXiv:1006.2231 [hep-ph]

57. A. Arhrib, M. Capdequi Peyranere, W. Hollik and G. Moultaka, Nucl. Phys. B 581, 34 (2000) [Erratum-ibid. 2004, 400 (2004)] [hep-ph/9912527] 